\title{
A current perspective on wound healing and tumour-induced angiogenesis
}

\author{
Jennifer A. Flegg* . Shakti N. Menon* . \\ Helen M. Byrne • D. L. Sean McElwain
}

Received: date / Accepted: date

\begin{abstract}
Angiogenesis, or capillary growth from pre-existing vasculature, is an essential component of several physiological processes, both vital and pathological. These include dermal wound healing and tumour growth, that together pose some of the most significant challenges to healthcare systems worldwide. Over the last few decades, mathematical modelling has proven to be a valuable tool for unravelling the complex network of interactions that underlie such processes. Moreover, theoretical frameworks that describe some of the mechanical and chemical aspects of angiogenesis inherent in wound healing and tumour growth have revealed intriguing similarities between the two processes. In this review, we highlight some of the significant contributions made by mathematical models of tumour-induced and wound healing angiogenesis, and illustrate how advances in each field have been made using insights from the other. We also detail some open problems that could be addressed through a combination of theoretical and experimental approaches.
\end{abstract}

Keywords Angiogenesis · Wound healing · Tumours · Mathematical modelling

Jennifer A. Flegg

School of Mathematics and Statistics, University of Melbourne, Melbourne, Australia Tel.: +61383447523

E-mail: jennifer.flegg@unimelb.edu.au

Shakti N. Menon

The Institute of Mathematical Sciences, CIT Campus, Taramani, Chennai 600 113, India

E-mail: shakti@imsc.res.in

Helen M. Byrne

Mathematical Institute, University of Oxford, Oxford, UK

E-mail: helen.byrne@maths.ox.ac.uk

D. L. Sean McElwain

Institute of Health and Biomedical Innovation and School of Mathematical Sciences, Queensland University of Technology, Brisbane, QLD 4001, Australia

E-mail: s.mcelwain@qut.edu.au 


\section{Introduction}

A wound is a breakdown in the protective function of the skin or underlying tissues. The failure of certain wounds to heal is a significant socioeconomic burden worldwide. In the United States alone, the total spending for all wound types is estimated to be in the range of $\$ 28.1$ to $\$ 96.8$ billion per annum (Nussbaum et al. 2018). Those who suffer from non-healing wounds experience considerable pain, immobility, and a decreased quality of life (Thackham et al., 2008). While non-healing wounds place a major burden on healthcare systems, the total costs associated with the treatment of cancers has an even greater impact: in the US alone it is projected to be $\$ 173$ billion per annum in 2020 (Mariotto et al. 2011). It has long been observed that there are similarities between the biological processes that underpin wound healing and tumour growth, and this has led to tumours being described as "wounds that do not heal" (Dvorak, 1986).

The wound healing process is complex, but is generally thought to consist of four overlapping and interconnected phases; haemeostasis, inflammation, proliferation and remodelling (for a detailed review of the biology of wound healing, see for example Flegg et al. (2015b); Singer and Clark (1999)). A schematic timeline of the four key stages of the wound healing process is displayed in Fig. 1. Briefly, in cutaneous (skin) wounds, haemostasis lasts a few hours after the initiation of a full-thickness wound (where both epidermis and dermis are damaged). Blood from the damaged capillaries carries platelets and fibrinogen into the wound site (Sheffield and Smith, 2002) leading to the creation of a fibrin clot that prevents further blood loss, and which acts as a provisional matrix for cell migration (Enoch and Leaper, 2005). The inflammation stage lasts for several days, and sees immune cells such as neutrophils and macrophages attracted to the wound site (by chemoattractants such as platelet derived growth factor (PDGF) and vascular en-

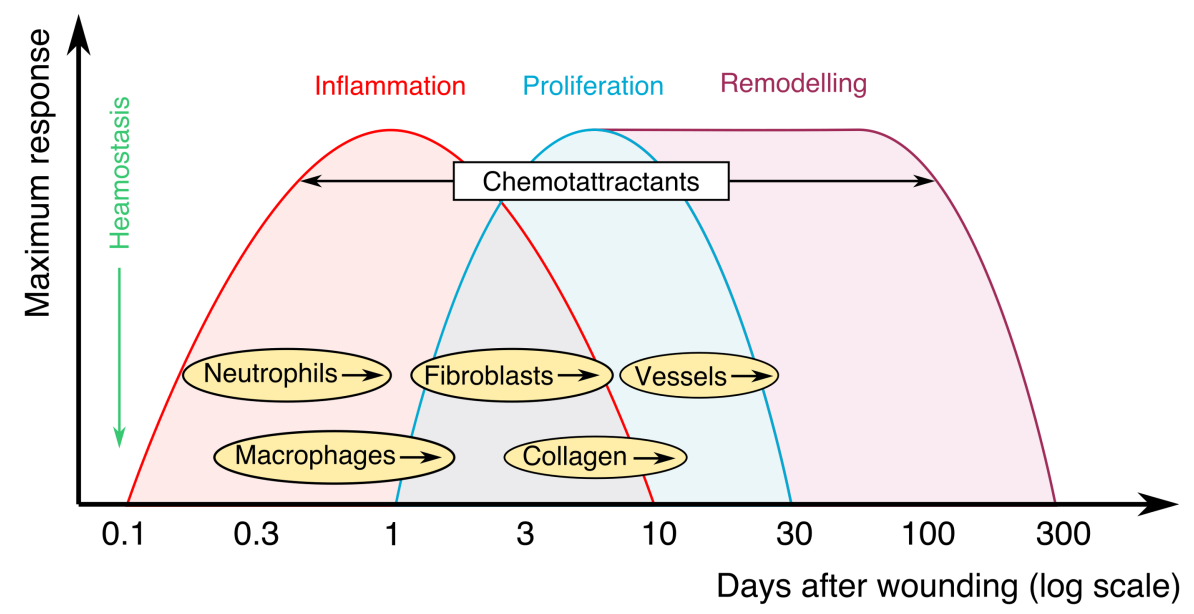

Fig. 1 Overview of the main stages of the wound healing process: haemostasis, inflammation, proliferation and remodelling. The key components of this process are labelled at the approximate times (post-injury) at which they first play a significant role. Figure adapted from Enoch et al. (2006). 
dothelial growth factor (VEGF) released earlier) in order to clear debris (Bellingan et al. 1996). During the proliferation phase, which lasts several weeks, fibroblasts are attracted to the wound site by chemoattractants and produce collagen, which is a major component of the new extracellular matrix that replaces the fibrin clot (Enoch et al. 2006). Angiogenesis, the process by which new blood vessels develop from preexisting vasculature (Bauer et al., 2005b), occurs largely during the proliferation stage. During angiogenesis, endothelial cells that line the blood vessels proliferate, rearrange and form new capillary buds; this process is stimulated by growth factors released during the earlier inflammation stage (Thackham et al. 2008). The formation of new blood vessels is critical to the healing process since oxygen, which blood vessels supply to the wound site, controls cellular activity (Tompach et al. 1997) and supports continued angiogenesis (Gordillo and Sen, 2003). During this stage, pericytes provide structural support to the existing blood vessels, and help regulate the proliferation and migration of endothelial cells (Bodnar et al. 2016). Angiogenesis is controlled by both chemical and mechanical cues in the wound site (Shiu et al. , 2005). The final remodeling phase lasts for several months (Sheffield and Smith, 2002) and sees the tensile strength across the wound increase (Bauer et al., 2005a).

In addition to playing a crucial role during wound healing, angiogenesis is also a critical component of tumour growth (Folkman, 1995). While there are similarities between tumour-induced and wound healing angiogenesis, there are also important differences. For example, while the need for oxygen and hypoxia (lack of oxygen) drive angiogenesis in both cases (Dvorak, 1986), the new vessel growth during wound healing is tightly regulated and organised whereas the network that results from tumour vessels is unorganized and becomes increasingly tortuous (Chaplain and Byrne, 1996; Forster et al. 2017). Mathematical models have provided insight into both tumour-induced and wound healing angiogenesis, and have generated theoretical predictions that have stimulated further biomedical research. In their 1996 paper, Chaplain and Byrne first noted that tumour growth and healing wounds could be modeled with similar mathematical frameworks (Chaplain and Byrne, 1996), an idea that we will explore throughout this review.

The aim of this paper is to highlight some of the main contributions of mathematical models of angiogenesis in the contexts of wound healing as well as tumour growth, and to outline how the evolution of each field has the potential to be informed by concurrent developments in the other. The paper is structured as follows: in the next section we compare and contrast wound healing angiogenesis with tumour-induced angiogenesis. We then highlight important contributions to the literature on mathematical models of wound healing and tumour-induced angiogenesis. Finally, we discuss some open problems in the field, before concluding with a discussion.

\section{Wound healing and tumour-induced angiogenesis: two sides of the same coin}

Generally speaking, angiogenesis is initiated in a wound healing or tumour context when cells in the vicinity of blood vessels are stimulated to release pro-angiogenic chemicals, such as VEGF, due to hypoxic conditions (Takenaga, 2011, Thackham et al. 2008). We note that while there are many isoforms of VEGF and other 


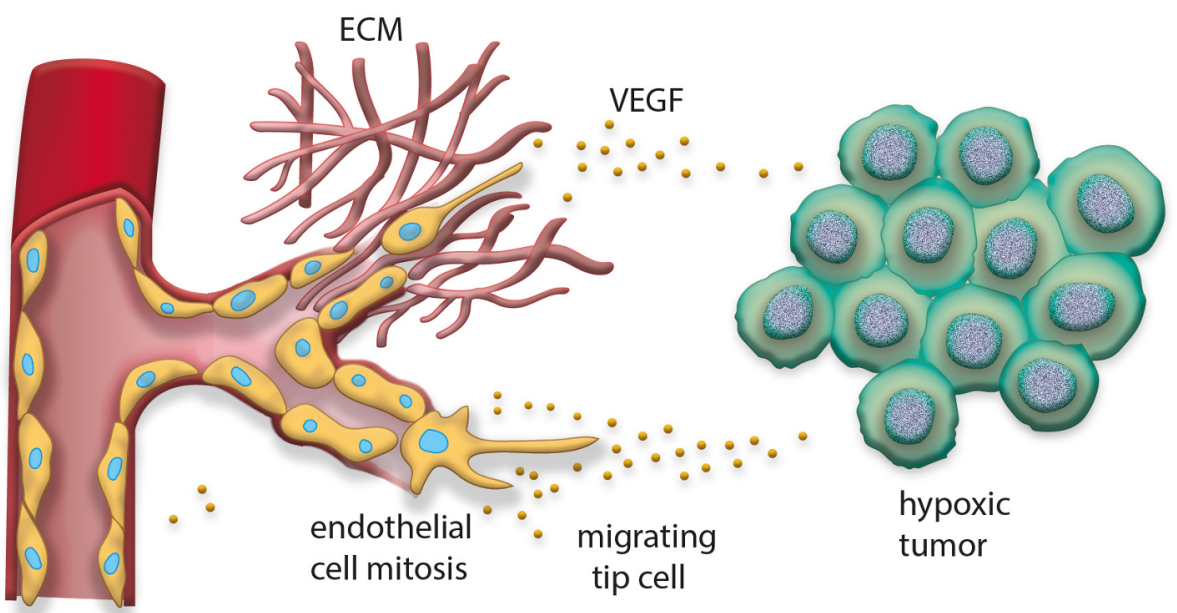

Fig. 2 Schematic illustration of events of angiogenesis (here shown for tumour-induced angiogenesis), modified from Bauer et al. (2007).

chemical stimulants involved in angiogenesis, this distinction is not crucial for the current discussion and indeed most existing mathematical models consider a single, generic angiogenic factor which can be viewed as a theoretical composite of the multiple angiogenic factors that act during angiogenesis. The release of these chemicals stimulates the degradation of the basement membrane that lines the blood vessels, leading to endothelial cells being able to break free of their parent vessel and migrate towards sources of pro-angiogenic chemicals (Bauer et al. 2005b). As illustrated in Fig. 2 these cells actively migrate through the extracellular matrix (ECM) by extending filopodia in the direction of the source of angiogenic stimuli and "crawling" towards it (Gerhardt et al., 2003). The activity of cells during angiogenesis is controlled largely by cell signalling, whereby receptors on the surface of the cell receive extracellular molecules that provide external signals to the cell to, for example, promote migration or produce a growth factor. The collective migration of endothelial cells results in the formation of a capillary bud from the parent blood vessel, led by a migrating tip cell (Adams and Alitalo, 2007). The extension of the capillary tip is facilitated by further migration of endothelial cells, as well as their proliferation which is largely confined to the region just behind the tip (see Fig. 2) (Gerhardt et al., 2003). After a sufficient degree of tip growth, tip-to-tip and tip-to-vessel joinings occur (referred to as "anastomoses"), which facilitates blood flow through the extended network (Mantzaris et al. 2004) and hence supply nutrients to the newly vascularised tissue. This process continues with the emergence of new tips and capillaries elongating to form an extensive vascular network that penetrates further into the stroma (the supportive framework for the surrounding tissue, including connective tissue, nerves, ducts etc).

Wound healing and tumour growth differ significantly in a number of ways, not least in terms of their physiological impact. However, the process of angiogenesis follows a similar course in both cancer and healing tissues, both of which are also characterised by the presence of a fibrin clot and inflammatory cells (Ribatti and Tamma, 2018). Additionally, cells in tumours and wounds express VEGF which 
increases vascular hyperpermeability, and thereby causes vessels to leak plasma fibrinogen (Dvorak, 2015). This stimulates angiogenesis and provides a matrix on which the vessels can develop (Ribatti and Tamma, 2018). Over time, the fibrin clot is degraded by inflammatory cells that are recruited into the tissue, and is subsequently replaced with a largely collagen-based matrix (Dvorak, 1986).

In wounds, the initial release of fibrinogen is a consequence of vessel injury, while in tumours it is driven by vascular hyperpermeability (Dvorak, 2015). Furthermore, VEGF production in wounds stops after the tissue is healed and hypoxic cues have been relieved, while in tumours these stimuli persist and promote further angiogenesis (Dvorak, 1986). The vessels associated with tumours are abnormally thick and leaky, while the cessation of VEGF signalling in wounds leads to a restoration of the normal basement membrane thickness and results in nonleaky vessels (Ribatti and Tamma, 2018). The hallmarks of tumour vasculature are poor blood flow and leakage, whereas mature vessels in successfully healed tissue are fully functional. While the initial fibrin clot in healed wounds will be completely degraded and replaced with a mature extracellular matrix (Thackham et al. 2008), leaky tumour blood vessels continue to supply the stroma with fibrinogen, leading to further fibrin clotting (Dvorak, 1986). In order to support the increased metabolic needs of a healing tissue, the vascular density in wounds is higher than that of normal tissue. Finally, excess vessels in wounds regress once healing is complete, resulting in a final vascular density similar to that of normal skin (DiPietro, 2016), which is not the case in tumours due to the persistence of the angiogenic stimulus.

\section{Advances in the modelling of tumour-induced and wound healing angiogenesis}

There have been numerous advances in the mathematical modelling of angiogenesis over the last 35 years, as illustrated in the timeline displayed in Fig. 3 . As a detailed description of all significant models of angiogenesis is beyond the scope of this review, we restrict attention to models that introduced new theoretical frameworks for investigating angiogenesis in the contexts of wound healing and tumour growth. For a recent review that systematically elucidates the principles underlying the development of reaction-advection-diffusion models for wound healing angiogenesis, see Flegg et al. (2015b). We also note that there have been several reviews of mathematical modelling of tumour-induced angiogenesis including Plank and Sleeman (2003b); Mantzaris et al. (2004); Chaplain et al. (2006), although there have been more recent developments not reviewed in those papers.

Chaplain and Byrne (1996) drew attention to the fact that the mathematical modelling of wound healing angiogenesis could benefit from insights gained from models of tumour-induced angiogenesis and vice-versa. Although a single mathematical model of angiogenesis would be unable to capture all relevant physiological details in each process, Chaplain and Byrne (1996) proposed a unifying framework that opened up new perspectives for the development of such models. In particular, they outlined an approach through which the steps involved in angiogenesis could be described through a system of partial differential equations (PDEs). As we shall discuss, there have been several subsequent models of wound healing angiogenesis that have incorporated ideas from models of tumour-induced angiogenesis, and 


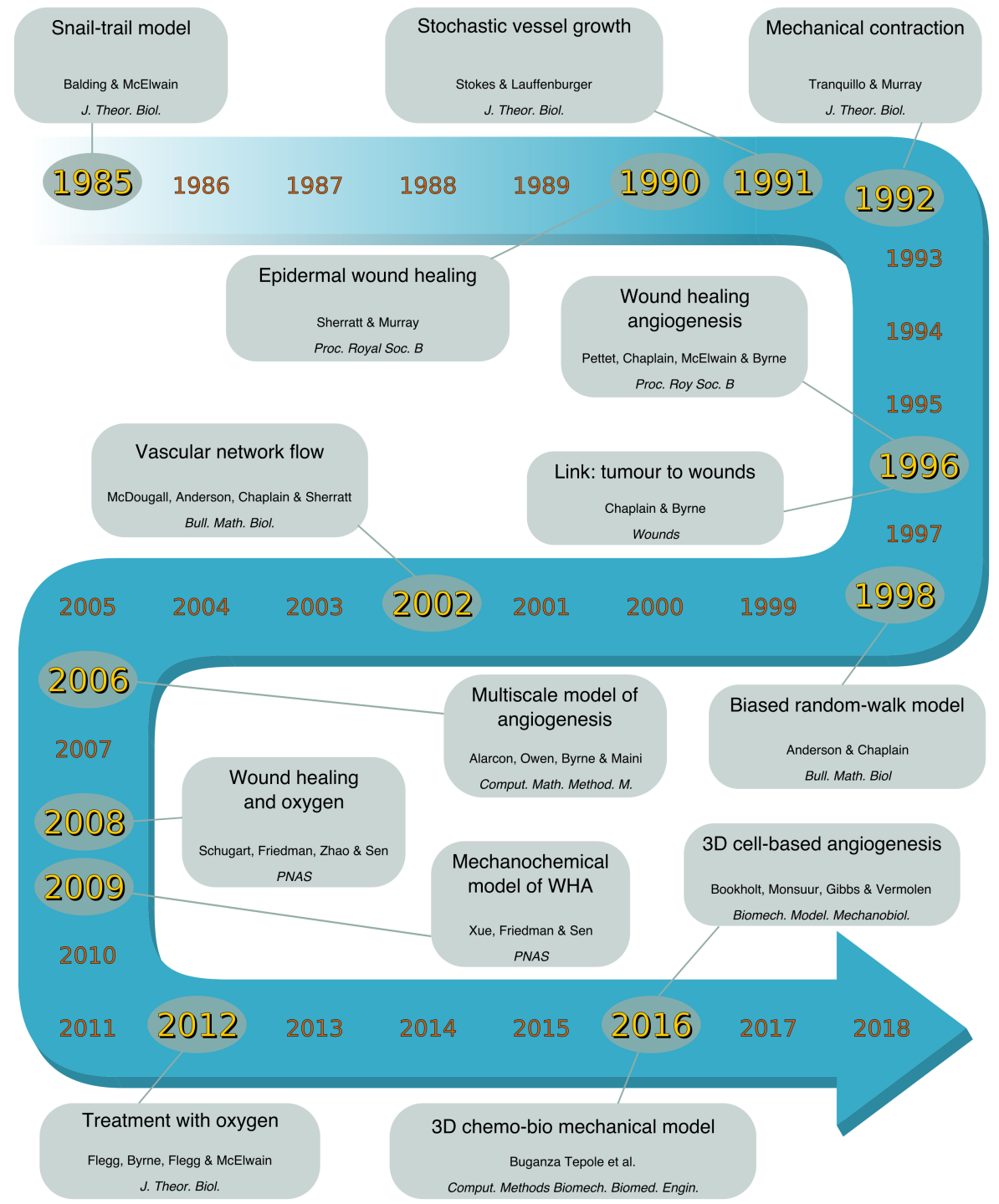

Fig. 3 Timeline of some of the significant works that triggered advances in the mathematical modelling of angiogenesis in the contexts of wound healing and tumour growth.

vice versa. In the following, we discuss some of the significant models of angiogenesis highlighted in Fig. 3 , and focus in particular on the novelty of the modelling framework in each case.

3.1 Early influential mathematical models

Some of the earliest theoretical frameworks developed to describe tumour-induced vascularization were the models of Saidel et al. (1976) and Liotta et al. (1977). 
Saidel et al. (1976) proposed a spatially-averaged model to describe tumour growth and vascularization, introducing different compartments to represent different cell types (e.g. proliferating tumour cells and tumour vascular cells). By contrast, Liotta et al. (1977) used what they referred to as a "diffusion model" to simulate the random motion of these quantities in space and time through a diffusion term. Using a diffusion-reaction model, Zawicki et al. (1981) proposed what we consider to be the first model for neovascularization in normal tissue, looking at vessels, tissue, and debris and comparing to their own experimental results. While these models provided a simple and robust approach for studying neovascularization, they did not explicitly consider the growth of capillary tips.

One of the first models to explicitly do so was proposed by Balding and McElwain (1985). As illustrated in Fig. 4 this model was motivated by a mathematical model for fungal growth developed by Edelstein (1982). Fungal hyphae are branched filaments that exhibit similar characteristics to capillaries and which, through a process similar to that observed during neovascularization, extend through growth at the tips. Edelstein (1982) described this mechanism using a model involving two dependent variables representing the hyphal and tip densities, respectively. The model explicitly accounts for an array of hyphal-tip interactions such as branching and anastomosis. Building upon this work, Balding and McElwain (1985) developed a one-dimensional (1-D) model that described tumour-induced capillary growth in terms of two continuous variables: the densities of the sprout tips and capillaries. In addition, they accounted for tip creation and anastomosis through a partial differential equation (PDE) that governs the evolution of capillary density. In contrast to Edelstein (1982), who assumed a constant tip extension rate, Balding and McElwain (1985) assumed that tips move via chemotaxis towards a source of Tumour Angiogenesis Factor (TAF). The resulting model, which comprises a system of coupled PDEs, described the "snail-trail" of capillaries that form behind the tips cells. They considered a case in which the source of TAF was instantaneously removed, and observed that while this causes angiogenic activity to cease within a few days, vascular decay occurred over a much longer time scale. These results were found to be consistent with independent experimental observations and, as we discuss below, the simplicity and generality of this modelling approach was highly influential on numerous subsequent models of angiogenesis.

The model of Balding and McElwain (1985) led to several subsequent studies that attempted to capture aspects of tumour-induced angiogenesis. The model developed by Chaplain and Stuart (1993) explicitly described the uptake of the TAF by proliferating endothelial cells, and this was generalized to assume that endothelial cells underwent random motion (modelled with a diffusion term) in a density-dependent manner and also included the effects of budding and anastomosis (Chaplain, 1995, 1996). A similar approach was employed by Orme and Chaplain (1996, 1997) who investigated the contribution of haptotaxis to the formation of capillary buds and the migration of sprouts towards the tumour source in both 1-D and 2-D geometries. This was extended by Anderson and Chaplain (1998b) to include chemotaxis towards TAF, in order to investigate why tips stop migrating in the absence of mitosis. A modified version of the model of Balding and McElwain (1985) was considered by Byrne and Chaplain (1995), who reproduced the experimentally-observed 'brush border' effect (Gimbrone Jr et al., 1974, Ausprunk and Folkman, 1977), where the frequency of branching increases in the vicinity of the tumour. More recently, Connor et al. (2015) extended the work of 


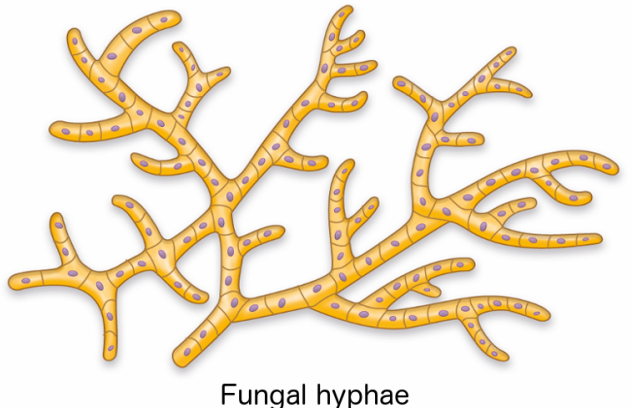

Fungal hyphae

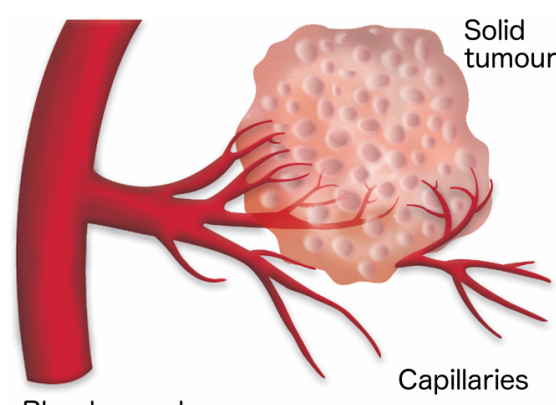

Blood vessel

Fig. 4 Schematics illustrating the similarities between the growth of fungal hyphae (left, modified from Campbell et al. (2009) ) and capillaries that extend away from the basement membranes of blood vessels towards a solid tumour (right, modified from Carmeliet (2000). Both processes lead to the development of a branched network that extends through growth at the tips. Balding and McElwain (1985) drew upon this similarity to develop the first mathematical model of tumour-induced angiogenesis, utilizing concepts from a previously developed model for fungal growth (Edelstein, 1982).

Balding and McElwain (1985) to develop a PDE model of angiogenesis, directed by two angiogenic factors, VEGF-A and basic fibroblast growth factor (bFGF).

Another influential mathematical model that was developed around this time was that of Sherratt and Murray (1990) who provided the first theoretical description of epidermal wound closure. This model assumed that the epidermis was sufficiently thin that the wound could be described over a 2-D domain. Under the assumption of radial symmetry, the model described the spatio-temporal distribution of cell density, which changes due to cell migration (described by a nonlinear diffusion term) and cell proliferation (described by a logistic growth term). The parameter $p$ controls the nature of the diffusive term that models the random motility of cells as linear $(p=0$, referred to as the Fisher or Kolmogorov-Petrovsky-Piskunov (KPP) equation or Fisher-KPP equation) or nonlinear $(p>0$, referred to as the porous Fisher equation). The model was extended to include the effect of a diffusible chemical that regulates cell proliferation, leading to a set of coupled PDEs. While this model did not explicitly describe angiogenesis, the idea of using the principle of conservation to develop coupled PDE models of wound healing was later extended to include angiogenesis (e.g. Pettet et al. (1996a); Olsen et al. (1998); Schugart et al. (2008); Flegg et al. (2012)).

\subsection{Stochastic models of angiogenesis}

While the model by Balding and McElwain (1985) captured a number of macroscopic features of tumour-angiogenesis, it did not consider the movement of individual capillary tips, or the conditions under which anastomosis of tips would occur. A probabilistic model of angiogenesis that took this into account was developed by Stokes and Lauffenburger (1991). Their model described bud formation from pre-existing parent vessels, and the subsequent migration of sprouts in the presence of a chemoattractant source (see Fig. 5). Each sprout $i$ has a density $\rho_{i}$ that varies as their tips extend away from the parent vessel. The evolution of 


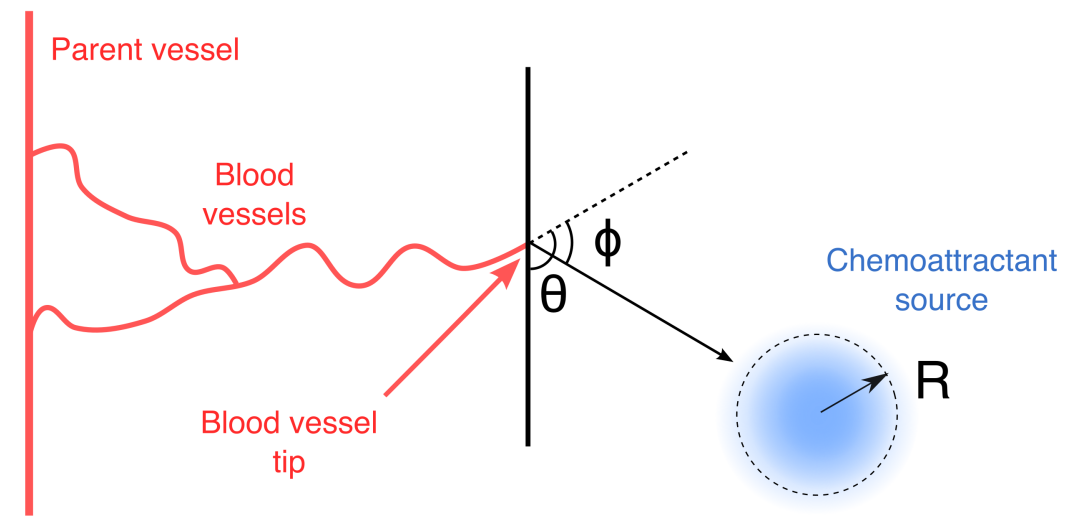

Fig. 5 Schematic illustrating the probabilistic model of Stokes and Lauffenburger (1991), which described capillary tip growth towards a chemoattractant source of radius $R$. In the displayed schematic, which is modified from the one in Stokes and Lauffenburger (1991), the leading cell of the blood vessel tip moves in a direction given by the angle $\theta$ from the vertical. The angle between the direction the cell moves and that of the chemoattractant is $\phi$. The leading cell uses membrane receptors to detect the direction of the chemoattractant source, and consequently the cell attempts to change its direction of motion by an angle $\phi$.

each $\rho_{i}$ was described using an ordinary differential equation that depended on the rate of proliferation of cells in the sprout, the rate at which the sprout elongates, the transfer of cells to the sprout from its parent vessel, and the transfer of cells from the sprout to any sprout that may branch away from it. When the density of a sprout exceeds a critical value $\rho_{\max }$, it was assumed that cells within that sprout stop proliferating, while if it drops below a value $\rho_{\min }$ the sprout cannot elongate further. The velocity $\mathbf{v}_{i}$ of each sprout tip was assumed to evolve via an Orstein-Uhlenbeck process, and was modelled using a stochastic ordinary differential equation that included a white noise process, a drift term for the bias of motion in the direction of the chemoattractant source, and a deterministic decay term. Furthermore, it was assumed that new tips may bud from an existing sprout $i$ if $\rho_{i}>\rho_{\text {min }}$, while if an existing sprout intersects with another, anastomosis occurs and $\mathbf{v}_{i}$ is set to zero. Results obtained using their model suggested that the directed growth of the network of sprouts required a moderate chemotactic response. The authors validated their model through a comparison with experimental data (Stokes et al., 1991), and concluded that an Orstein-Uhlenbeck process provides a reasonable description of tip migration. This modelling framework hence offered a robust approach to describe the conditions that facilitate vessel formation. Only a few subsequent studies have used stochastic differential equations to model vascular growth induced by the presence of a tumour such as the work of Capasso and Morale (2009). Thus, this approach remains a relatively under-utilised mathematical tool in this field.

\subsection{Mechanochemical models}

The first mathematical model that integrated mechanical aspects into a model of wound closure was developed by Tranquillo and Murray (1992). They considered 


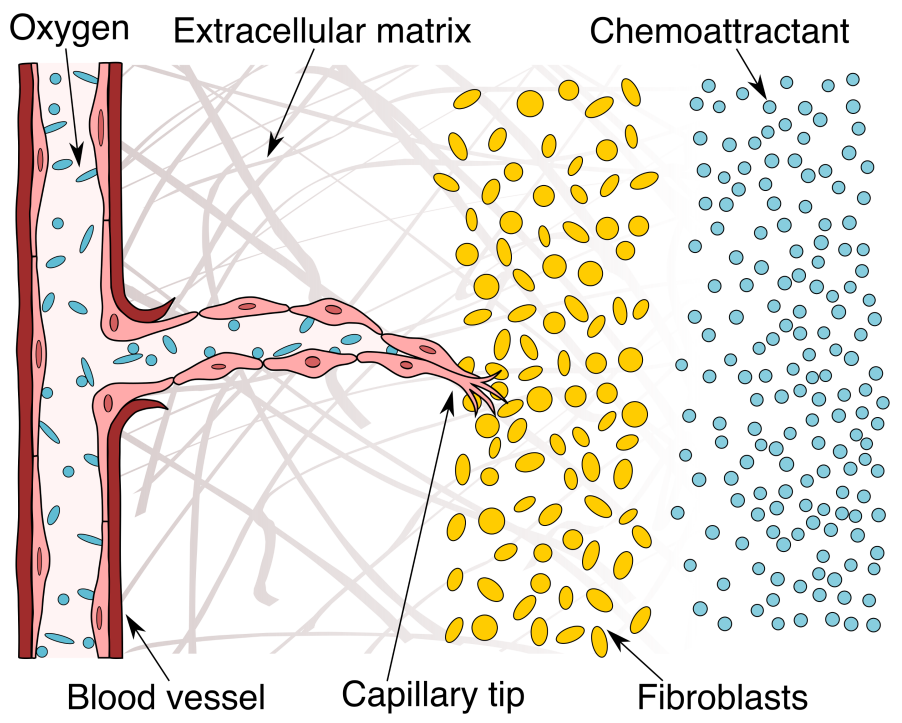

Fig. 6 Schematic illustrating the components of the model of angiogenesis developed by Pettet et al. (1996a), which considered the role of oxygen in the formation of new capillaries, as well as the proliferation of fibroblasts.

the interaction of fibroblasts and ECM during healing as well as the displacement of their composite. They employed a force balance for the cell/ECM composite that included the traction forces that fibroblast cells exert on the ECM. Central to this model was the idea that the cell/ECM composite could be described as a linear viscoelastic solid.

While the work of Tranquillo and Murray (1992) neglected consideration of angiogenesis, the pioneering theoretical framework proposed in their paper inspired numerous subsequent mechanochemical models. For instance, Olsen and coworkers built upon this modelling approach to describe the role of myofibroblasts in fibrocontractive diseases (Olsen et al., 1995), the growth factor mediated responses that are associated with fibro-proliferative disorders such as keloid and hypertrophic scars (Olsen et al., 1996), as well as the process through which collagen fibres of the ECM are realigned by invading cells that exhibit directed migration, or haptotaxis (Olsen et al. 1998). Importantly in the context of this review paper, Olsen et al. (1997) included the concepts of haptotaxis and haptokinesis (random migration mediated by the ECM) in a model of the spatio-temporal evolution of the endothelial cell density during wound healing angiogenesis (without considering cell alignment). Another contemporaneous work that incorporated the reorganization of the ECM was by Dallon et al. (1999), who modelled discrete fibroblast cells in a wound and allowed fibroblasts to produce collagen fibres and change the direction of the fibres to follow the local ECM already laid down. Later work built on the idea of modelling the ECM as a viscoelastic material, including the model of wound angiogenesis developed by Xue et al. (2009), which we will discuss in the following section.

In the context of tumour angiogeneis, one of the first mechanochemical models was developed by Holmes and Sleeman (2000). They built upon the model of Chap- 
lain and Stuart (1993), and used the frameworks of Murray and Oster (1984) and Manoussaki et al. (1996) to incorporate cellular traction and to describe the ECM as a 2-D viscoelastic material. In addition to considering haptotaxis and chemotaxis of endothelial cells, their model described a mechanism by which endothelial cells reoriented the collagen fibres of the ECM through traction forces, leading to deformations of the ECM and a subsequent feedback on the direction of cell migration. More recently, Zheng et al. (2013) developed a mechanochemical model of angiogenesis that considered the regulating mechanisms of VEGF during initial vessel outgrowth, sprout extension and vessel maturation.

\subsection{Modelling the role of oxygen}

One of the first models of wound healing angiogenesis that considered the role of oxygen in mediating chemoattractant production, as well as the proliferation of fibroblasts, was developed by Pettet et al. (1996a). This model incorporated the 'snail trail' mechanism of Balding and McElwain (1985) and described the wound healing process as a set of six coupled PDEs that modelled the spatiotemporal evolution of capillary tips, capillary sprouts, ECM, oxygen, fibroblasts and chemoattractants (as illustrated in Fig. 6). Simulation results agreed well with experimental results in that low oxygen regions were needed in the wound to drive healing and that excess levels lead to the cessation of angiogenesis.

As blood supply to vascular beds is essential for successful wound healing, situations where blood flow is impeded (for instance due to arterial diseases) can greatly disrupt the wound healing process. To this end, Xue et al. (2009) developed a model (outlined in Fig. 7) to investigate the impact of ischemic conditions on wound closure. While the role of tissue oxygenation on dermal wound healing had been previously studied (Pettet et al., 1996a; Schugart et al., 2008), the model by Xue et al. (2009) described the ECM as a growing viscoelastic material (similar to Tranquillo and Murray (1992)), and was hence one of the first mechanochemical models of wound angiogenesis. Using this model, which comprised a system of PDEs, they examined the role that ischemic conditions play on wound closure.

Subsequently, Flegg and coworkers developed a set of mathematical models using reaction-advection-diffusion equations inspired by works such as Pettet et al. (1996b a) and Byrne et al. (2000) to consider non-healing, chronic wounds and investigated how certain treatments might stimulate healing in an otherwise nonhealing wound. This included the effect of changing oxygen supply/demand (Flegg et al. 2012), supplemental oxygen through hyperbaric oxygen therapy (Flegg et al. 2009, 2010), compression bandages (Flegg et al., 2015a), and the interaction of dermal and epidermal cells (Menon et al. 2012).

\subsection{Discrete models of angiogenesis}

While most models of wound healing angiogenesis developed during the 1990s used continuum approaches, several contemporaneous discrete models of tumourinduced angiogenesis attempted to capture behaviour at the level of individual cells. Most notably, the model of Anderson and Chaplain (1998a) described the 
formation of a network of capillary sprouts in response to TAF. This approach differed from that of Stokes and Lauffenburger (1991) in that the development of the model involved the discretization of a continuum model for the rate of change of endothelial cell density. The continuum model, which also captured the 'brush border' effect, consisted of a system of PDEs that described the chemotactic response of endothelial cells to cytokines, as well as their directed migration into the ECM via haptotaxis. More recently, Jain and Jackson (2013) modelled the chemotactic sensitivity of the leading tip cells in the brush border to VEGF stimulus.

The modelling framework introduced by Anderson and Chaplain (1998a) led to several subsequent models of angiogenesis including those by McDougall et al. (2002, 2006); Alarcón et al. (2006) and Owen et al. (2009). McDougall et al. (2002) extended the approach of Anderson and Chaplain (1998a) to incorporate fluid flow through the vascular network. Specifically, they computed the flow rate of a fluid across each element of the growing capillary network using Poiseuille's law. This modelling approach allowed the authors to investigate the conditions under which injected chemotherapeutic drugs may bypass the tumour as a consequence of the highly interconnected nature of the vascular network. Their results suggested that the structure of the vasculature in the vicinity of the tumour needs to be taken into account when developing chemotherapeutic strategies. This approach was later extended to describe flow in 3-D networks (Stephanou et al., 2005), where it was demonstrated that drug uptake could be enhanced by random removal of vessels. While these studies assumed that the spatial and temporal evolution of the vascular network proceeded in a manner independent of the fluid flow, McDougall et al. (2006) considered a dynamic adaptive tumour-induced angiogenesis (DATIA) model, which incorporated flow-mediated vessel adaptation and capillary remodelling. They predicted that the blood plasma viscosity does not play an appreciable role in determining the final network structure, whereas decreasing intravascular pressure would reduce the density of the resulting network. The role of flow-mediated vessel adaptation was also considered by Alarcón et al. (2006) and Owen et al. (2009).

In contrast to the model of Anderson and Chaplain (1998a), which focused on the development of capillaries from pre-existing sprouts, the model of Levine et al. (2000, 2001b) investigated the changes within the existing vessels that are necessary for the initiation of angiogenesis. Their model consisted of a coupled system of ordinary and partial differential equations, and used the theory of reinforced random walks to describe the motion of endothelial cells as well as a MichaelisMenten mechanism to describe the production of proteolytic enzymes. This work was extended in Levine et al. (2001a) to incorporate the migration of endothelial cells through the ECM towards the tumour. The idea of modelling tumour angiogenesis as a reinforced random walk was also employed by Sleeman and Wallis (2002) and Plank and Sleeman (2003a) to describe the chemotactic and haptotactic response of endothelial cells towards a source of TAF, and their modelling approach was subsequently extended to describe the motion of cells (Plank and Sleeman, 2004). 


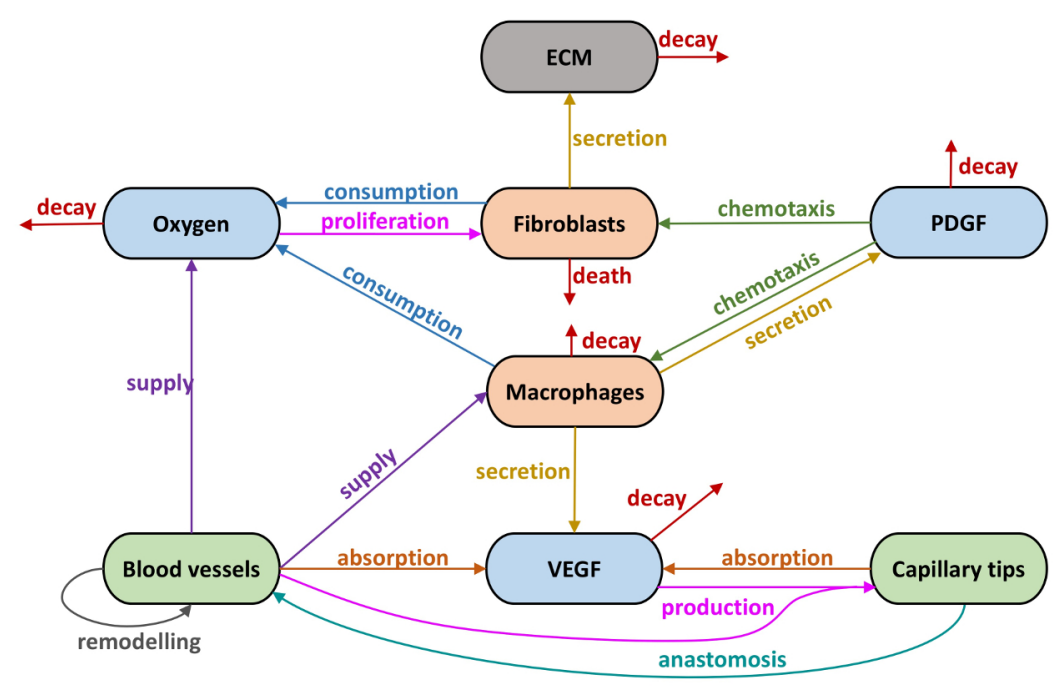

Fig. 7 Schematic illustrating the various components of the mechanochemical model of dermal wound healing developed by Xue et al. (2009). The labelled arrows indicate the interactions between the components of the model.

3.6 Multiscale models of angiogenesis

In addition to continuum and discrete modelling approaches, each of which have their advantages, several multiscale models of angiogenesis have integrated modelling techniques across several spatial scales. The first multiscale models of tumour angiogenesis were those developed by Alarcón et al. (2006) and McDougall et al. (2006) who integrated a model of individual endothelial cell migration with a network flow model. A similar framework was used by Owen et al. (2009), who developed a multiscale model of vascular tissue growth that incorporated blood flow and vessel remodelling. Macklin et al. (2009) developed a multiscale model of an expanding tumour that combined the discrete modelling of vessel growth from Anderson and Chaplain (1998a) and McDougall et al. (2006).

Around the same time, Bauer et al. (2007) proposed a multiscale model of tumour-induced angiogenesis, that described the dynamics of the extracellular environment using a PDE, and the interactions between endothelial cells and the stroma using the cellular Potts model. The processes captured at the extracellular level included the release of VEGF from hypoxic tumours, as well as the binding of VEGF to endothelial cells through receptors. At the cellular level the model included migration and proliferation of endothelial cells through the ECM, as well as the degradation of the ECM and VEGF-stimulated mitosis of cells. This model was later extended to incorporate stalk cell chemotaxis and cell recruitment (Bauer et al., 2009). Subsequent multiscale models of tumour angiogenesis include those by Frieboes et al. (2010), who investigated the conditions that impact the morphology of an invading tumour, and Cai et al. (2011) who incorporated the role of blood perfusion. 
3.7 Three-dimensional models of angiogenesis

In recent years, there have been efforts to extend existing mathematical models into 3-D. For example, Milde et al. (2008) and Shirinifard et al. (2009), followed a similar approach to that of Bauer et al. (2007) to develop 3-D multiscale models, using the cellular Potts model, in order to simulate the coupled cellular behaviour during tumour-induced angiogenesis. More recently, Bookholt et al. (2016) modelled angiogenesis in vitro using a cell-based model in 3-D, where the tip cells are the leading cells of the new blood vessel and the stalk cells follow behind the tip into the fibrin matrix. This model accounted for several mechanisms, including adhesion to the basement membrane, contact mechanics between cells, haptotaxis and chemotaxis. Around the same time, Buganza Tepole and Kuhl (2016) developed a model to identify the chemical, biological, and mechanical mechanisms of scar formation in the remodeling phase of wound healing. They considered the evolution of a generic inflammatory chemical signal, fibroblasts, collagen and the mechanical properties of tissue in a 3 -D wound space. Other recent advances include the development of open software for simulating vascularised tissues in 3-D (Grogan et al. 2017), a tool that has been applied to model corneal angiogenesis (Grogan et al., 2018). Grogan et al. (2017) found that using a 3-D geometry is important in recreating vascular patterning consistent with experiments. Contemporaneously, Perfahl et al. (2017) developed an agent-based model in a 3-D multiscale model of vasculogenesis, the de novo formation of blood vessels from endothelial progenitor cells, generating simulations that were consistent with both in-vitro and in-vivo experiments.

In this section, we have highlighted some of the contributions to the field of mathematical modelling of angiogenesis in wound healing and tumours. It is beyond the scope of this paper to review every contribution made to this field. The more recent models have drawn heavily on the earlier works and have culminated in complicated models in 3-D. While 3-D models are a natural extension of the existing models that have been reviewed in this section and have the benefit of being more realistic biologically, there are drawbacks of higher dimensional models. These include the requirement of more sophisticated techniques to approximate the numerical solution. Moreover, the required computational time increases with the complexity of structure and/or geometry of the process being modelled, and is hence significantly greater for higher dimensional systems. As we shall discuss in the "Open Problems" section, this leads to difficulties in determining the optimal values of the model parameters.

\section{Open Problems}

As detailed in the previous section, recent decades have seen significant advances in the mathematical modelling of angiogenesis in the contexts of wound healing and tumour growth. However, there remain unresolved questions, including those listed below.

\section{Model parameter estimation}

In order for any mathematical model to have predictive capabilities, it is important that the values of the associated model parameters are appropriately 
sourced. Historically, formal statistical methods have not been employed to estimate parameter values for mathematical models of tumour growth or wound healing, although this has changed recently (see, for example, Flegg et al. (2015b); Jin et al. (2018)). An added difficulty here is that it is typically not possible to infer all wound healing (or tumour) model parameters from a single experiment (laboratory or clinical). Consequently, parameter values are often estimated from a variety of sources. For example, Sherratt and Murray (1990) compared their model predictions to in vivo rabbit studies while Tranquillo and Murray (1992) used data from in vivo rat experiments. More recently, Jin et al. (2018) formally fitted model results to in vitro cell experiments, while Flegg et al. (2015b) fitted to data from human clinical trials. Data from each of these sources are typically associated with different statistical observational models and error structures. An open problem in this field is to use modern applied statistical techniques, which have gained favour in other areas of mathematical biology, to infer the model parameter values in models of wound healing and tumour-induced angiogenesis. These include Bayesian approaches, which allow different error structures from varying data sources to be naturally accounted for (Gelman et al., 2013). To do this, there needs to be access to good quality data for parameter estimation (and model validation), which is an open problem that mathematicians and experimentalists need to work closely together to address, for example using mathematical models to guide experimental design. For model parameter estimation, the time it takes to simulate the model for a single set of parameter values can be a limiting factor in the quality of the parameter estimation and should be considered during the model development stage. There are also related questions around model selection and model comparison. That is, when comparing competing models, which one/s would the data best support?

The available data leads to questions about the level of detail that one would need to include in the model. That is, does one require resolution at the level of individual blood vessels or individual endothelial cells, or would a course-grained tissue-scale model suffice? Alternatively, one may ask whether a combination of scales might be appropriate? Indeed, there are mathematical techniques that have been applied in an angiogenesis context that allow the relationship between models at different spatial scales to be combined, see for example Pillay et al. (2017, 2018). However, we note that even when experimental data is available, there is no guarantee it would be sufficient to parameterise the model and there may, in addition, be significant parameter identifiability issues that could stem from the quality and quantity of the data.

\section{Treatment of wounds and tumours}

In addition to gaining insights into the mechanisms that underpin angiogenesis in wounds, mathematical modelling can also be used to assess novel treatment strategies, such as hyperbaric oxygen therapy and alternative designs for compression bandages (Flegg et al. 2015b, 2009, 2010), and there is further work that can be done in this research area. This includes investigating how negative pressure and electric fields, both of which may affect angiogenesis, might aid in the treatment of wounds. Treatments at the cellular scale in the contexts of both wounds and tumours can also be further investigated from a theoretical point of view, including the role of immune cells in regulating angiogenesis, 
and the manipulation of VEGF or delta-notch signalling pathways to promote or inhibit angiogenesis (Carmeliet, 2003, Bentley et al., 2008).

Angiogenesis in other contexts

Just as the mathematical modelling of wound healing angiogenesis has drawn upon work in tumour-induced modelling, there are other contexts in which angiogenesis is relevant. These include eye disease such as age-related macular degeneration and their treatment (Witmer et al. 2003), which has been modelled mathematically (Hutton-Smith et al. | 2018, 2016), menstruation, arthritis, atherosclerosis and embryogenesis. It is possible that these fields could benefit from advances made in the context of modelling tumour/wound angiogenesis (or vice versa). There have been mathematical models of angiogenesis, vasculogensis and vascular remodelling that are not specific to wound healing or tumour angiogenesis (see, for example Gödde and Kurz (2001); Secomb et al. (2013)) of which researchers in both fields should be aware. Peirce (2008) provides an excellent review of mathematical modelling of angiogenesis across various contexts.

Addressing unanswered questions relating to angiogenesis

Mathematical modelling can help answer questions related to the angiogenesis process. These include, but are not limited to: do blood vessels collapse due to cell apoptosis or cell migration away from the vessel? Why are some capillaries larger in diameter than others? Does internal blood flow drive angiogenic growth? While existing multiscale models of vascular tumour growth, for example Alarcón et al. (2006), account for changes in vessel radius and vessel pruning in response to both biochemical and mechanical stimuli, it is likely that a discrete mathematical model framework would be helpful in addressing some of these questions at the cellular level. These include the cell-based simulation software, Chaste (Cancer, Heart and Soft Tissue Environment), see https://www.cs.ox.ac.uk/chaste/, and Microvessel Chaste which can be used to explicitly model vascularised tissues (Grogan et al., 2017). Recently developed experimental techniques have led to the availability of data that can be used to parameterise such mathematical models of 3-D microvessel formation (see for example Jeon et al. (2014)).

\section{Discussion}

In this paper, we have highlighted how progress in the mathematical modelling of wound healing angiogenesis and tumour-induced angiogenesis have been possible through a cross-pollination of ideas over the last 35 years, and how further advances may be acheived in each area. In summarising the research area, we have identified several issues where more work is required to move this field forward, including the estimation of model parameters, extending these ideas to model angiogenesis in other contexts, and the use of modelling to address questions related to the treatment of abnormal wounds. The aim of the paper was not to systematically cover an exhaustive list of studies that have modelled wound healing or tumour-induced angiogenesis. Rather our goal was to comprehensively summarise 
the overlapping fields of mathematical modelling of wound healing and tumourinduced angiogenesis.

With large amounts of biological data becoming available at fine-scale spatiotemporal resolutions, there is an increased need for mathematical models to keep pace with experimental observations, as well as the parameter inference methods being adopted. The analysis of detailed models of increased complexity will require significant computational resources, as well as a broad range of expertise. Hence it will be important to involve a suitably skilled interdisciplinary team (including, for instance, biologists, applied mathematicians, clinicians, physicists, computer scientists and applied statisticians) to address such problems. Ultimately, this requires a common language for communication between these different scientists; we hope this review can contribute to developing that common language by highlighting the progress of the field to date.

Acknowledgements JAF would like to acknowledge funding from the Australian Research Council (DE160100227) that supported this work. SNM is supported by the IMSc Complex Systems Project (12th Plan). We would also like to thank Rocio A. Mince for help with the illustrated schematics.

\section{References}

Adams RH, Alitalo K (2007) Molecular regulation of angiogenesis and lymphangiogenesis. Nat Rev Mol Cell Bio 8(6):464, doi 10.1038/nrm2183

Alarcón T, Owen MR, Byrne HM, Maini PK (2006) Multiscale modelling of tumour growth and therapy: the influence of vessel normalisation on chemotherapy. Comput Math Methods Med 7(2-3):85-119, doi:10.1080/10273660600968994

Anderson ARA, Chaplain MAJ (1998a) Continuous and discrete mathematical models of tumor-induced angiogenesis. Bull Math Biol 60(5):857-899, doi: $10.1006 /$ bulm.1998.0042

Anderson ARA, Chaplain MAJ (1998b) A mathematical model for capillary network formation in the absence of endothelial cell proliferation. Appl Math Lett 11(3):109-114, doi:10.1016/S0893-9659(98)00041-X

Ausprunk DH, Folkman J (1977) Migration and proliferation of endothelial cells in preformed and newly formed blood vessels during tumor angiogenesis. Microvasc Res 14(1):53-65, doi:10.1016/0026-2862(77)90141-8

Balding D, McElwain DLS (1985) A mathematical model of tumour-induced capillary growth. J Theor Biol 114(1):53-73, doi:10.1016/S0022-5193(85)80255-1

Bauer AL, Jackson TL, Jiang Y (2007) A cell-based model exhibiting branching and anastomosis during tumor-induced angiogenesis. Biophys J 92(9):31053121, doi $10.1529 /$ biophysj.106.101501

Bauer AL, Jackson TL, Jiang Y (2009) Topography of extracellular matrix mediates vascular morphogenesis and migration speeds in angiogenesis. PLoS Comp Biol 5(7):e1000445, doi 10.1371/journal.pcbi.1000445

Bauer SM, Bauer RJ, Liu ZJ, Chen H, Goldstein LJ, Velazquez OC (2005a) Vascular endothelial growth factor-c promotes vasculogenesis, angiogenesis, and collagen constriction in three-dimensional collagen gels. J Vasc Surg 41(4):699-707, doi: $10.1016 /$ j.jvs.2005.01.015 
Bauer SM, Bauer RJ, Velazquez OC (2005b) Angiogenesis, vasculogenesis, and induction of healing in chronic wounds. Vasc Endovascular Surg 39(4):293-306, doi: $10.1177 / 153857440503900401$

Bellingan GJ, Caldwell H, Howie SEM, Dransfield I, Haslett C (1996) In vivo fate of the inflammatory macrophage during the resolution of inflammation. J Immunol 157:2577-2585

Bentley K, Gerhardt H, Bates PA (2008) Agent-based simulation of notchmediated tip cell selection in angiogenic sprout initialisation. J Theor Biol 250(1):25-36, doi 10.1016/j.jtbi.2007.09.015

Bodnar RJ, Satish L, Yates CC, Wells A (2016) Pericytes: A newly recognized player in wound healing. Wound Repair Regen 24(2):204-214, doi $10.1111 /$ wrr. 12415

Bookholt F, Monsuur H, Gibbs S, Vermolen F (2016) Mathematical modelling of angiogenesis using continuous cell-based models. Biomech Model Mechanobiol 15(6):1577-1600, doi:10.1007/s10237-016-0784-3

Buganza Tepole A, Kuhl E (2016) Computational modeling of chemo-bio-mechanical coupling: A systems-biology approach toward wound healing. Comput Methods Biomech Biomed Eng 19(1):13-30, doi:10.1080/10255842.2014.980821

Byrne HM, Chaplain MAJ (1995) Mathematical models for tumour angiogenesis: Numerical simulations and nonlinear wave solutions. Bull Math Biol 57(3):461486, doi:10.1016/S0092-8240(05)81778-1

Byrne HM, Chaplain MAJ, Evans DL, Hopkinson I (2000) Mathematical modelling of angiogenesis in wound healing: Comparison of theory and experiment. Comput Math Methods Med 2(3):175-197, doi:10.1080/10273660008833045

Cai Y, Xu S, Wu J, Long Q (2011) Coupled modelling of tumour angiogenesis, tumour growth and blood perfusion. J Theor Biol 279(1):90-101, doi:10.1016/j.jtbi.2011.02.017

Campbell NA, Reece JB, Taylor MR, Simon EJ, Dickey JL (2009) Biology: Concepts \& connections. Pearson/Benjamin Cummings, California

Capasso V, Morale D (2009) Stochastic modelling of tumour-induced angiogenesis. J Math Biol 58(1-2):219-233, doi:10.1007/s00285-008-0193-z

Carmeliet P (2000) Mechanisms of angiogenesis and arteriogenesis. Nat Med $6(4): 389$, doi: $10.1038 / 74651$

Carmeliet P (2003) Angiogenesis in health and disease. Nature medicine 9(6):653, doi: $10.1038 / \mathrm{nm} 0603-653$

Chaplain MAJ (1995) The mathematical modelling of tumour angiogenesis and invasion. Acta Biotheor 43(4):387-402, doi 10.1007/BF00713561

Chaplain MAJ (1996) Avascular growth, angiogenesis and vascular growth in solid tumours: The mathematical modelling of the stages of tumour development. Math Comput Model 23(6):47-87, doi:10.1016/0895-7177(96)00019-2

Chaplain MAJ, Byrne HM (1996) Mathematical modelling of wound healing and tumour growth: Two sides of the same coin. Wounds 8(2):42-48

Chaplain MAJ, Stuart AM (1993) A model mechanism for the chemotactic response of endothelial cells to tumour angiogenesis factor. Math Med Biol 10(3):149-168, doi 10.1093/imammb/10.3.149

Chaplain MAJ, McDougall SR, Anderson ARA (2006) Mathematical modeling of tumor-induced angiogenesis. Annu Rev Biomed Eng 8:233-257, doi:10.1146/annurev.bioeng.8.061505.095807 
Connor AJ, Nowak RP, Lorenzon E, Thomas M, Herting F, Hoert S, Quaiser T, Shochat E, Pitt-Francis J, Cooper J, et al. (2015) An integrated approach to quantitative modelling in angiogenesis research. J R Soc Interface 12(110):20150546, doi:10.1098/rsif.2015.0546

Dallon JC, Sherratt JA, Maini PK (1999) Mathematical modelling of extracellular matrix dynamics using discrete cells: fiber orientation and tissue regeneration. J Theor Biol 199(4):449-471, doi 10.1006/jtbi.1999.0971

DiPietro LA (2016) Angiogenesis and wound repair: When enough is enough. J Leukocyte Biol 100(5):979-984, doi:10.1189/jlb.4MR0316-102R

Dvorak HF (1986) Tumors: Wounds that do not heal. similarities between tumor stroma generation and wound healing. New Engl J Med 315(26):1650-1659, doi $10.1056 /$ NEJM198612253152606

Dvorak HF (2015) Tumors: Wounds that do not heal-redux. Cancer Immunol Res 3(1):1-11, doi 10.1158/2326-6066.CIR-14-0209

Edelstein L (1982) The propagation of fungal colonies: A model for tissue growth. J Theor Biol 98(4):679-701, doi:10.1016/0022-5193(82)90146-1

Enoch S, Leaper DJ (2005) Basic science of wound healing. Surgery 23(2):37-42, doi:10.1383/surg.23.2.37.60352

Enoch S, Grey JE, Harding KG (2006) Recent advances and emerging treatments. BMJ 332(7547):962-965, doi 10.1136/bmj.332.7547.962

Flegg JA, McElwain DL, Byrne HM, Turner IW (2009) A three species model to simulate application of hyperbaric oxygen therapy to chronic wounds. PLoS Comp Biol 5(7):e1000451, doi:10.1371/journal.pcbi.1000451

Flegg JA, Byrne HM, McElwain DLS (2010) Mathematical model of hyperbaric oxygen therapy applied to chronic diabetic wounds. Bull Math Biol 72(7):18671891, doi $10.1007 / \mathrm{s} 11538-010-9514-7$

Flegg JA, Byrne HM, Flegg MB, McElwain DLS (2012) Wound healing angiogenesis: The clinical implications of a simple mathematical model. J Theor Biol 300:309-316, doi:10.1016/j.jtbi.2012.01.043

Flegg JA, Kasza J, Darby I, Weller CD (2015a) Healing of venous ulcers using compression therapy: Predictions of a mathematical model. J Theor Biol 379:19, doi:10.1016/j.jtbi.2015.04.028

Flegg JA, Menon SN, Maini PK, McElwain DLS (2015b) On the mathematical modeling of wound healing angiogenesis in skin as a reaction-transport process. Front Physiol 6:262, doi 10.3389/fphys.2015.00262

Folkman J (1995) Angiogenesis in cancer, vascular, rheumatoid and other disease. Nature Med 1(1):27, doi 10.1038/nm0195-27

Forster JC, Harriss-Phillips WM, Douglass MJJ, Bezak E (2017) A review of the development of tumor vasculature and its effects on the tumor microenvironment. Hypoxia 5:21, doi:10.2147/HP.S133231

Frieboes HB, Jin F, Chuang YL, Wise SM, Lowengrub JS, Cristini V (2010) Threedimensional multispecies nonlinear tumor growth-II: Tumor invasion and angiogenesis. J Theor Biol 264(4):1254-1278, doi 10.1016/j.jtbi.2010.02.036

Gelman A, Carlin JB, Stern HS, Dunson DB, Vehtari A, Rubin DB (2013) Bayesian data analysis. Chapman and Hall/CRC

Gerhardt H, Golding M, Fruttiger M, Ruhrberg C, Lundkvist A, Abramsson A, Jeltsch M, Mitchell C, Alitalo K, Shima D, et al. (2003) VEGF guides angiogenic sprouting utilizing endothelial tip cell filopodia. J Cell Biol 161(6):1163-1177, doi: $10.1083 /$ jcb.200302047 
Gimbrone Jr MA, Cotran RS, Leapman SB, Folkman J (1974) Tumor growth and neovascularization: An experimental model using the rabbit cornea. J Natl Cancer Inst 52(2):413-427, doi $10.1093 /$ jnci/52.2.413

Gödde R, Kurz H (2001) Structural and biophysical simulation of angiogenesis and vascular remodeling. Dev Dyn 220(4):387-401, doi 10.1002/dvdy.1118

Gordillo GM, Sen CK (2003) Revisiting the essential role of oxygen in wound healing. Am J Surg 186(3):259-263, doi 10.1016/S0002-9610(03)00211-3

Grogan JA, Connor AJ, Markelc B, Muschel RJ, Maini PK, Byrne HM, PittFrancis JM (2017) Microvessel chaste: an open library for spatial modeling of vascularized tissues. Biophys J 112(9):1767-1772, doi $10.1016 /$ j.bpj.2017.03.036

Grogan JA, Connor AJ, Pitt-Francis JM, Maini PK, Byrne HM (2018) The importance of geometry in the corneal micropocket angiogenesis assay. PLoS Comp Biol 14(3):e1006049, doi 10.1371/journal.pcbi.1006049

Holmes MJ, Sleeman BD (2000) A mathematical model of tumour angiogenesis incorporating cellular traction and viscoelastic effects. J Theor Biol 202(2):95112, doi $10.1006 /$ jtbi.1999.1038

Hutton-Smith LA, Gaffney EA, Byrne HM, Maini PK, Schwab D, Mazer NA (2016) A mechanistic model of the intravitreal pharmacokinetics of large molecules and the pharmacodynamic suppression of ocular vascular endothelial growth factor levels by ranibizumab in patients with neovascular age-related macular degeneration. Mol Pharm 13(9):2941-2950, doi:10.1021/acs.molpharmaceut.5b00849

Hutton-Smith LA, Gaffney EA, Byrne HM, Caruso A, Maini PK, Mazer NA (2018) Theoretical insights into the retinal dynamics of vascular endothelial growth factor in patients treated with ranibizumab, based on an ocular pharmacokinetic/pharmacodynamic model. Mol Pharm 15(7):2770-2784, doi:10.1021/acs.molpharmaceut.8b00280

Jain H, Jackson T (2013) A hybrid model of the role of VEGF binding in endothelial cell migration and capillary formation. Frontiers in Oncology 3:102, doi 10.3389 /fonc. 2013.00102

Jeon JS, Bersini S, Whisler JA, Chen MB, Dubini G, Charest JL, Moretti M, Kamm RD (2014) Generation of 3d functional microvascular networks with human mesenchymal stem cells in microfluidic systems. Integr Biol 6(5):555563 , doi $10.1039 / \mathrm{c} 3 \mathrm{ib} 40267 \mathrm{c}$

Jin W, Lo KY, Chou SE, McCue SW, Simpson MJ (2018) The role of initial geometry in experimental models of wound closing. Chem Eng Sci 179:221-226, doi: $10.1016 /$ j.ces.2018.01.004

Levine HA, Sleeman BD, Nilsen-Hamilton M (2000) A mathematical model for the roles of pericytes and macrophages in the initiation of angiogenesis. i. the role of protease inhibitors in preventing angiogenesis. Math Biosci 168(1):77-115, doi:10.1016/S0025-5564(00)00034-1

Levine HA, Pamuk S, Sleeman BD, Nilsen-Hamilton M (2001a) Mathematical modeling of capillary formation and development in tumor angiogenesis: Penetration into the stroma. Bull Math Biol 63(5):801-863, doi: $10.1006 /$ bulm.2001.0240

Levine HA, Sleeman BD, Nilsen-Hamilton M (2001b) Mathematical modeling of the onset of capillary formation initiating angiogenesis. J Math Biol 42(3):195238, doi $10.1007 / \mathrm{s} 002850000037$ 
Liotta LA, Saidel GM, Kleinerman J (1977) Diffusion model of tumor vascularization and growth. Bull Math Biol 39(1):117-128, doi:10.1016/S00928240(77)80040-2

Macklin P, McDougall S, Anderson AR, Chaplain MAJ, Cristini V, Lowengrub J (2009) Multiscale modelling and nonlinear simulation of vascular tumour growth. J Math Biol 58(4-5):765-798, doi:10.1007/s00285-008-0216-9

Manoussaki D, Lubkin SR, Vemon RB, Murray JD (1996) A mechanical model for the formation of vascular networks in vitro. Acta Biotheor 44(3-4):271-282, doi:10.1007/BF00046533

Mantzaris NV, Webb S, Othmer HG (2004) Mathematical modeling of tumorinduced angiogenesis. J Math Biol 49(2):111-187, doi:10.1007/s00285-003-02622

Mariotto AB, Robin Yabroff K, Shao Y, Feuer EJ, Brown ML (2011) Projections of the cost of cancer care in the united states: 2010-2020. J Natl Cancer Inst 103(2):117-128, doi:10.1093/jnci/djq495

McDougall SR, Anderson ARA, Chaplain MAJ, Sherratt JA (2002) Mathematical modelling of flow through vascular networks: implications for tumourinduced angiogenesis and chemotherapy strategies. Bull Math Biol 64(4):673702, doi $10.1006 /$ bulm.2002.0293

McDougall SR, Anderson ARA, Chaplain MAJ (2006) Mathematical modelling of dynamic adaptive tumour-induced angiogenesis: Clinical implications and therapeutic targeting strategies. J Theor Biol 241(3):564-589, doi $10.1016 /$ j.jtbi.2005.12.022

Menon SN, Flegg JA, McCue SW, Schugart RC, Dawson RA, McElwain DS (2012) Modelling the interaction of keratinocytes and fibroblasts during normal and abnormal wound healing processes. Proc Roy Soc B 279(1741):3329-3338, doi $10.1098 /$ rspb.2012.0319

Milde F, Bergdorf M, Koumoutsakos P (2008) A hybrid model for threedimensional simulations of sprouting angiogenesis. Biophys J 95(7):3146-3160, doi:10.1529/biophysj.107.124511

Murray JD, Oster GF (1984) Cell traction models for generating pattern and form in morphogenesis. J Math Biol 19(3):265-279, doi:10.1007/BF00277099

Nussbaum SR, Carter MJ, Fife CE, DaVanzo J, Haught R, Nusgart M, Cartwright D (2018) An economic evaluation of the impact, cost, and medicare policy implications of chronic nonhealing wounds. Value Health 21(1):27-32, doi:10.1016/j.jval.2017.07.007

Olsen L, Sherratt JA, Maini PK (1995) A mechanochemical model for adult dermal wound contraction and the permanence of the contracted tissue displacement profile. J Theor Biol 177(2):113-128, doi 10.1006/jtbi.1995.0230

Olsen L, Sherratt JA, Maini PK (1996) A mathematical model for fibro-proliferative wound healing disorders. Bull Math Biol 58(4):787-808, doi: $10.1007 / \mathrm{BF} 02459482$

Olsen L, Sherratt JA, Maini PK, Arnold F (1997) A mathematical model for the capillary endothelial cell-extracellular matrix interactions in wound-healing angiogenesis. Math Med Biol 14(4):261-281, doi 10.1093/imammb/14.4.261

Olsen L, Maini PK, Sherratt JA, Marchant B (1998) Simple modelling of extracellular matrix alignment in dermal wound healing i. cell flux induced alignment. J Theor Med 1(3):175-192, doi:10.1080/10273669808833018 
Orme ME, Chaplain MAJ (1996) A mathematical model of the first steps of tumour-related angiogenesis: Capillary sprout formation and secondary branching. Math Med Biol 13(2):73-98, doi:10.1093/imammb/13.2.73

Orme ME, Chaplain MAJ (1997) Two-dimensional models of tumour angiogenesis and anti-angiogenesis strategies. Math Med Biol 14(3):189-205, doi: $10.1093 /$ imammb/14.3.189

Owen MR, Alarcón T, Maini PK, Byrne HM (2009) Angiogenesis and vascular remodelling in normal and cancerous tissues. J Math Biol 58(4-5):689, doi: $10.1007 / \mathrm{s} 00285-008-0213-\mathrm{z}$

Peirce SM (2008) Computational and mathematical modeling of angiogenesis. Microcirculation 15(8):739-751, doi 10.1080/10739680802220331

Perfahl H, Hughes BD, Alarcón T, Maini PK, Lloyd MC, Reuss M, Byrne HM (2017) 3d hybrid modelling of vascular network formation. J Theor Biol 414:254268, doi $10.1016 /$ j.jtbi.2016.11.013

Pettet G, Chaplain MAJ, McElwain DLS, Byrne HM (1996a) On the role of angiogenesis in wound healing. Proc Roy Soc B 263(1376):1487-1493, doi $10.1098 / \mathrm{rspb} .1996 .0217$

Pettet GJ, Byrne HM, McElwain DLS, Norbury J (1996b) A model of woundhealing angiogenesis in soft tissue. Math Biosci 136(1):35-63, doi:10.1016/00255564(96)00044-2

Pillay S, Byrne HM, Maini PK (2017) Modeling angiogenesis: A discrete to continuum description. Phys Rev E 95(1):012410, doi 10.1103/PhysRevE.95.012410

Pillay S, Byrne HM, Maini PK (2018) The impact of exclusion processes on angiogenesis models. J Math Biol 77(6-7):1721-1759, doi:10.1007/s00285-018-1214-1

Plank MJ, Sleeman BD (2003a) A reinforced random walk model of tumour angiogenesis and anti-angiogenic strategies. Math Med Biol 20(2):135-181, doi $10.1093 /$ imammb/20.2.135

Plank MJ, Sleeman BD (2003b) Tumour-induced angiogenesis: A review. J Theor Med 5(3-4):137-153, doi:10.1080/10273360410001700843

Plank MJ, Sleeman BD (2004) Lattice and non-lattice models of tumour angiogenesis. Bull Math Biol 66(6):1785-1819, doi:10.1016/j.bulm.2004.04.001

Ribatti D, Tamma R (2018) A revisited concept. tumors: Wounds that do not heal. Crit Rev Oncol Hemat 128:65-69, doi:10.1016/j.critrevonc.2018.05.016

Saidel GM, Liotta LA, Kleinerman J (1976) System dynamics of a metastatic process from an implanted tumor. J Theor Biol 56(2):417-434, doi:10.1016/S00225193(76)80083-5

Schugart RC, Friedman A, Zhao R, Sen CK (2008) Wound angiogenesis as a function of tissue oxygen tension: A mathematical model. Proc Natl Acad Sci USA 105(7):2628-2633, doi:10.1073/pnas.0711642105

Secomb TW, Alberding JP, Hsu R, Dewhirst MW, Pries AR (2013) Angiogenesis: An adaptive dynamic biological patterning problem. PLoS Comp Biol 9(3):e1002983, doi 10.1371/journal.pcbi.1002983

Sheffield PJ, Smith APS (2002) Physiological and pharmacological basis of hyperbaric oxygen therapy. In: Bakker DJ, Cramer FS (eds) Hyperbaric Surgery, Best Publishing Company, chap 4, pp 63-109

Sherratt JA, Murray JD (1990) Models of epidermal wound healing. Proc Roy Soc B 241(1300):29-36, doi 10.1098/rspb.1990.0061

Shirinifard A, Gens JS, Zaitlen BL, Popławski NJ, Swat M, Glazier JA (2009) 3d multi-cell simulation of tumor growth and angiogenesis. PloS One 4(10):e7190, 
doi:10.1371/journal.pone.0007190

Shiu YT, Weiss JA, Hoying JB, Iwamoto MN, Joung IS, Quam CT (2005) The role of mechanical stresses in angiogenesis. Critical reviews in biomedical engineering 33(5):431-510, doi 10.1615/CritRevBiomedEng.v33.i5.10

Singer AJ, Clark RAF (1999) Cutaneous wound healing. New Engl J Med 341(10):738-746, doi:10.1056/NEJM199909023411006

Sleeman B, Wallis IP (2002) Tumour induced angiogenesis as a reinforced random walk: Modelling capillary network formation without endothelial cell proliferation. Math Comput Model 36(3):339-358, doi:10.1016/S0895-7177(02)00129-2

Stephanou A, McDougall SR, Anderson ARA, Chaplain MAJ (2005) Mathematical modelling of flow in $2 \mathrm{~d}$ and $3 \mathrm{~d}$ vascular networks: Applications to anti-angiogenic and chemotherapeutic drug strategies. Math Comput Model 41(10):1137-1156, doi: $10.1016 /$ j.mcm.2005.05.008

Stokes CL, Lauffenburger DA (1991) Analysis of the roles of microvessel endothelial cell random motility and chemotaxis in angiogenesis. J Theor Biol 152(3):377403, doi 10.1016/S0022-5193(05)80201-2

Stokes CL, Lauffenburger DA, Williams SK (1991) Migration of individual microvessel endothelial cells: Stochastic model and parameter measurement. J Cell Sci 99(2):419-430

Takenaga K (2011) Angiogenic signaling aberrantly induced by tumor hypoxia. Frontiers in Bioscience 16:31-48, doi $10.2741 / 3674$

Thackham JA, McElwain DLS, Long RJ (2008) The use of hyperbaric oxygen therapy to treat chronic wounds: A review. Wound Repair Regen 16(3):321330, doi $10.1111 / \mathrm{j} .1524-475 X .2008 .00372 . x$

Tompach PC, Lew D, Stoll JL (1997) Cell response to hyperbaric oxygen treatment. Int J Oral Max Surg 26(2):82-86, doi 10.1016/S0901-5027(05)80632-0

Tranquillo RT, Murray JD (1992) Continuum model of fibroblast-driven wound contraction: Inflammation-mediation. J Theor Biol 158(2):135-172, doi: 10.1016/S0022-5193(05)80715-5

Witmer AN, Vrensen GFJM, Van Noorden CJF, Schlingemann RO (2003) Vascular endothelial growth factors and angiogenesis in eye disease. Prog Retin Eye Res 22(1):1-29, doi:10.1016/S1350-9462(02)00043-5

Xue C, Friedman A, Sen CK (2009) A mathematical model of ischemic cutaneous wounds. Proc Natl Acad Sci USA 106(39):16782-16787, doi: $10.1073 /$ pnas.0909115106

Zawicki DF, Jain RK, Schmid-Schoenbein GW, Chien S (1981) Dynamics of neovascularization in normal tissue. Microvasc Res 21(1):27-47, doi:10.1016/00262862(81)90003-0

Zheng X, Koh GY, Jackson T (2013) A continuous model of angiogenesis: initiation, extension, and maturation of new blood vessels modulated by vascular endothelial growth factor, angiopoietins, platelet-derived growth factor-b, and pericytes. Discrete and Continuous Dynamical Systems-Series B 18(4):11091154, doi $10.3934 /$ dcdsb.2013.18.1109 